\title{
Analytic Model of Microcantilevers as Low Frequency Generator
}

\author{
Sandeep Arya, Saleem Khan, and Parveen Lehana \\ Department of Physics \& Electronics, University of Jammu, Jammu 180006, India \\ Correspondence should be addressed to Parveen Lehana; parveenlehanajournals@gmail.com
}

Received 3 January 2014; Accepted 18 March 2014; Published 25 May 2014

Academic Editor: Nikos D. Lagaros

Copyright (C) 2014 Sandeep Arya et al. This is an open access article distributed under the Creative Commons Attribution License, which permits unrestricted use, distribution, and reproduction in any medium, provided the original work is properly cited.

\begin{abstract}
Finite element method (FEM) based simulation has been carried out, and an analytic model of microcantilevers using piezoelectric excitations is proposed. The model is based on the type of the selected material and geometry of the structure. The investigations are carried out with rectangular microcantilevers using silicon as the substrate. The high frequency analytic signals are applied to the input piezoelectric electrodes, and the resultant signal generated at the output piezoelectric electrode is recorded and analyzed. The analysis of the results showed that the proposed system is capable of generating a low frequency signal. Two microcantilevers with different dimensional aspects are used, and the results verified the application of microcantilever array as a low frequency signal generator.
\end{abstract}

\section{Introduction}

In spite of several applications of MEMS based materials in almost every field of science and technology, the applications involved in the bending of the free end of microcantilever beam that is parallel to the substrate bring the platform for new microdevices as sensors [1]. A sensor is a detector that can sense a specific phenomenon like changes in temperature, mass, or surface stress [2,3]. Microcantilevers based sensors work on the principle of converting mechanical energy that arises due to the deformation or deflection of the micromachined components into the desired read-out [4]. Thus, the cantilever is a mechanically sensitive tool that detects a specific sensing material loaded at the cantilever surface [5]. The vibration characteristics of a cantilever should be well identified in a proper design. The stretching or bending causes stiffness to the vibrating structure which results in the variation of mode shapes [6]. The role of piezoelectric material in sensor applications is significant. A piezoelectric sensor acquires a charge while being compressed and shows transducer effect between mechanical and electrical energy forms. In microcantilevers, a piezoelectric material observes some stiffness while bending due to the change in electric field [2, 7-9]. In addition to sensors, piezoelectric cantilevers can also be used as actuators [10]. Piezoelectric cantilevers can be analyzed under two actuation modes: the static and the dynamic modes. The static mode measures the bending of the cantilever beam while the dynamic mode measures the resonance of the beam [11, 12]. Different physical, chemical, and biosensing techniques had been reported by several researchers [13-17].

In this regard, this paper presents a finite element model to analyze the response of piezoelectric microcantilever as a low frequency generator. A thin layer of lead zirconate titanate (PZT-4D) was integrated on a rectangular silicon substrate, and two active metallic layers of aluminium in the form of beam are mounted whose one end is kept fixed and another is free to vibrate. COMSOL, a commercial finite element analysis tool, was used to develop and design a finite element model of the microcantilever.

\section{Mathematical Analysis}

The cantilever exhibits elastic property as that of a spring; hence, a cantilever supports the equation of motion for a spring and is given by

$$
m \frac{d^{2} x}{d t^{2}}+c \frac{d x}{d t}+k x=f(t),
$$

where $m$ is the mass of the beam, $c$ is the damping constant, $k$ is the spring constant, $x$ is the displacement, $d x / d t$ is the velocity, $d^{2} x / d t^{2}$ is the acceleration, and $f(t)$ is the forcing function. 
The natural frequency for a cantilever beam with no load is given as

$$
f_{n}=\frac{1}{2 \pi} \sqrt{\frac{k}{m}} .
$$

For a given finite load, damping is present. Under such conditions, equation of motion becomes

$$
\frac{d^{2} x}{d t^{2}}+c f^{\prime} \frac{d x}{d t}+f^{\prime 2} x=0
$$

where the zero on the right-hand side signifies the absence of excitation force and the value for the damping constant $c$ can be calculated as

$$
c=\frac{c_{1}}{2 \sqrt{k m}}
$$

where $c_{1}$ is the mass distribution function of the beam. Thus, the first mode natural frequency becomes

$$
f^{\prime 2} \propto \frac{E I / L^{3}}{L A \rho} .
$$

In the above equation, the numerator designates the damping constant $k$, whereas the denominator is related to the mass of the beam. Similarly, the $i$ th mode resonance frequency [18] of the beam can be calculated as

$$
f_{i}^{2} \propto\left(\frac{\alpha_{i}}{L}\right)^{4} \frac{E I}{\rho w t} .
$$

Here, $\rho$ is the density of material, $L$ is the length of the beam, $\alpha_{i}$ is the deflection angle at that instant, and $w$ and $t$ are the width and thickness of the beam, respectively.

For a rectangular beam, $I=w t^{3} / 12$, and hence, (6) becomes

$$
f_{i}^{2} \propto\left(\frac{\alpha_{i}}{L}\right)^{4} \frac{E t^{2}}{12 \rho} .
$$

Moreover, the spring constant $k$ of the cantilever is given by

$$
k=\frac{F}{\delta} \Longrightarrow \delta=\frac{F}{k}
$$

where spring constant $k$ is also termed as the stiffness (resistance to bending deformation) of a cantilever beam and the value for $k$ is given by

$$
k=\frac{3 E I}{L^{3}} .
$$

Figure 1 shows the rectangular beam under two conditions, that is, in a static position without stress and in a deflected position under some finite applied stress or pressure.

Hence, from (8), the deflection $\delta$ of the free end of the beam is given as

$$
\delta=\frac{F L^{3}}{3 E I}
$$

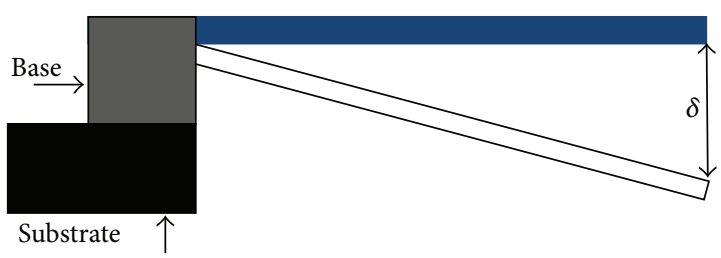

FIgURE 1: Static and deflected positions of a rectangular cantilever beam.

where $F$ is the force perpendicular to the cantilever end, $E$ is the Young modulus of elasticity, and $I$ is the function of beam width. The deflection in beam is due to the difference in stress on the top and bottom surfaces of the cantilever beam, and this difference can be calculated using Stoney's equation given as

$$
\frac{1}{R}=6 \frac{1-v}{E t^{2}}\left(\Delta \sigma_{1}-\Delta \sigma_{2}\right)
$$

where $R$ is the radius of curvature of microcantilever beam due to the stress on top and bottom surfaces, $v$ is Poisson's ratio of the material, $t$ is the thickness of the beam, and $\Delta \sigma_{1}$ and $\Delta \sigma_{2}$ are stresses that act on the top and bottom surfaces of the beam [19]. The deflection of such a beam can be estimated by the following relation:

$$
\frac{1}{R}=\frac{2 \delta}{L^{2}}
$$

\section{Piezoelectricity}

In this section, the finite element equations for piezoelectricity theory and its numerical approximation are presented. In piezoelectricity, an electric potential gradient causes deformation. Piezoelectricity is the interaction between linear elasticity equations and electrostatic charge equations by means of electric constants $[15,16]$. Let us consider a cantilever beam having a length $L$; then, the stress-charge form of the equations is given by

$$
\begin{gathered}
\varepsilon=S^{E} \sigma+d_{t} E^{\prime}, \\
D=d \sigma+\eta E^{\prime},
\end{gathered}
$$

where $\varepsilon$ is the strain vector, $S^{E}$ is the compliance or elasticity matrix, $\sigma$ is the stress vector, $d$ is the piezoelectric coupling coefficient matrix, $d_{t}$ is the transpose of the piezoelectric coupling coefficient matrix, $E^{\prime}$ is the electric field vector, $D$ is the electric displacement vector, and $\eta$ is the electric permittivity matrix [17]. The stress-charge equation is significant for finite element analysis due to its involvement in the conversion of mechanical strain into electrical power. In spite of the coupling matrix, the piezoelectric model also depends on the elasticity matrix and relative permittivity equations. Elasticity matrix is required to evaluate the elastic constants to transform the $\langle 100\rangle$ axes of the material into the axes of the substrate orientation. Elasticity is the relationship between 
stress and strain. Hooke's law describes the relationship between stress $(\sigma)$ and strain $(\varepsilon)$ and is given by

$$
\begin{aligned}
& \sigma=C \varepsilon, \\
& \varepsilon=S \sigma,
\end{aligned}
$$

where $C$ is the mechanical stiffness and $S$ is the compliance. The mechanical stiffness can be designated as a single value of Young's Modulus E. Hence, Hooke's law can also be written as

$$
\sigma=E \varepsilon
$$

The elasticity matrix for isotropic materials can be expressed as

$$
\begin{aligned}
S^{E}=\frac{E}{(1+\nu)(1-2 \nu)} & \\
& \times\left\{\begin{array}{cccccc}
1-\nu & \nu & \nu & 0 & 0 & 0 \\
\nu & 1-\nu & \nu & 0 & 0 & 0 \\
\nu & v & 1-\nu & 0 & 0 & 0 \\
0 & 0 & 0 & 1-2 \nu & 0 & 0 \\
0 & 0 & 0 & 0 & 1-2 \nu & 0 \\
0 & 0 & 0 & 0 & 0 & 1-2 \nu
\end{array}\right\},
\end{aligned}
$$

where $E$ is Young's modulus and $v$ is Poisson's ratio [18]. Relative permittivity, also known as dielectric constant, is the ratio of the permittivity of a specific material to that of free space or vacuum. Permittivity is the property of a medium that affects the magnitude of force between twopoint charges.

\section{Stress-Strain Relation}

Stress-strain relationship indicates the material property at a specific point on the system in any orientation. The material is generally isotropic as the properties of a material are stable in every plane [20]. However, there are some materials whose properties are orientation dependent and generally known as anisotropic materials. These materials follow Hooke's law. For a three-dimensional plane, the strain-stress relations for elastic materials are given by

$$
\left[\begin{array}{c}
\varepsilon_{1} \\
\varepsilon_{2} \\
\varepsilon_{3} \\
\varepsilon_{23} \\
\varepsilon_{13} \\
\varepsilon_{12}
\end{array}\right]=\left[\begin{array}{cccccc}
C_{11} & C_{12} & C_{13} & 0 & 0 & 0 \\
C_{12} & C_{22} & C_{23} & 0 & 0 & 0 \\
C_{13} & C_{23} & C_{33} & 0 & 0 & 0 \\
0 & 0 & 0 & C_{44} & 0 & 0 \\
0 & 0 & 0 & 0 & C_{55} & 0 \\
0 & 0 & 0 & 0 & 0 & C_{66}
\end{array}\right]\left[\begin{array}{c}
\sigma_{1} \\
\sigma_{2} \\
\sigma_{3} \\
\sigma_{23} \\
\sigma_{13} \\
\sigma_{12}
\end{array}\right],
$$

where the elements $C_{i j}$ are given by

$$
\begin{array}{lll}
C_{11}=\frac{1}{E_{11}}, & C_{12}=-\frac{v_{21}}{E_{22}}, & C_{13}=-\frac{v_{31}}{E_{32}}, \\
C_{22}=\frac{1}{E_{22}}, & C_{23}=-\frac{v_{32}}{E_{33}}, & C_{33}=\frac{1}{E_{33}}, \\
C_{44}=\frac{1}{G_{23}}, & C_{55}=\frac{1}{G_{13}}, & C_{66}=\frac{1}{G_{12}} .
\end{array}
$$

$E_{11}, E_{22}$, and $E_{33}$ denote Young's modulus in the 3dimensional plane; $G_{12}, G_{23}$, and $G_{13}$ represent the shear modulus; and $v_{21}, v_{31}$, and $v_{32}$ indicate Poisson's ratio.

\section{Compatibility Equations}

Compatibility equations rely on the statement, "If a body is incessant before deformation, it should remain incessant after deformation." Hence, there should be no fracture or break in the body due to deformation. Also, the overlapping should be avoided [19]. For three-dimensional structures, there are six compatibility equations as follows:

$$
\begin{gathered}
\frac{\partial^{2} \varepsilon_{x x}}{\partial y^{2}}+\frac{\partial^{2} \varepsilon_{y y}}{\partial x^{2}}=\frac{\partial^{2} \varepsilon_{x y}}{\partial x \partial y}, \\
\frac{\partial^{2} \varepsilon_{y y}}{\partial z^{2}}+\frac{\partial^{2} \varepsilon_{z z}}{\partial y^{2}}=\frac{\partial^{2} \varepsilon_{y z}}{\partial y \partial z}, \\
\frac{\partial^{2} \varepsilon_{z z}}{\partial x^{2}}+\frac{\partial^{2} \varepsilon_{x x}}{\partial z^{2}}=\frac{\partial^{2} \varepsilon_{z x}}{\partial x \partial z} \\
\frac{1}{2} \frac{\partial}{\partial x}\left(\frac{\partial \varepsilon_{x y}}{\partial z}-\frac{\partial \varepsilon_{y z}}{\partial x}+\frac{\partial \varepsilon_{z x}}{\partial y}\right)=\frac{\partial^{2} \varepsilon_{x x}}{\partial y \partial z} \\
\frac{1}{2} \frac{\partial}{\partial y}\left(\frac{\partial \varepsilon_{x y}}{\partial z}+\frac{\partial \varepsilon_{y z}}{\partial x}-\frac{\partial \varepsilon_{z x}}{\partial y}\right)=\frac{\partial^{2} \varepsilon_{y y}}{\partial z \partial x} \\
\frac{1}{2} \frac{\partial}{\partial z}\left(-\frac{\partial \varepsilon_{x y}}{\partial z}+\frac{\partial \varepsilon_{y z}}{\partial x}+\frac{\partial \varepsilon_{z x}}{\partial y}\right)=\frac{\partial^{2} \varepsilon_{z z}}{\partial x \partial y} .
\end{gathered}
$$

The piezoelectric material also depends on other factors that are equally responsible for the successful design of a microcantilever. These factors are elasticity matrix $\left(S^{E}\right)$, coupling matrix $(d)$, and relative permittivity $(\eta)$. The piezoelectric constants used in the design are as follows:

$$
S^{E}=\left(\begin{array}{cccccc}
S_{11} & S_{12} & S_{13} & S_{14} & 0 & 0 \\
S_{12} & S_{11} & S_{13} & -S_{14} & 0 & 0 \\
S_{13} & S_{13} & S_{33} & 0 & 0 & 0 \\
S_{14} & -S_{14} & 0 & S_{44} & 0 & 0 \\
0 & 0 & 0 & 0 & S_{44} & S_{14} \\
0 & 0 & 0 & 0 & S_{14} & \frac{\left(S_{11}-S_{12}\right)}{2}
\end{array}\right)
$$

The permittivity and the piezoelectric coupling matrices can be written as

$$
\begin{aligned}
& \eta=\left(\begin{array}{ccc}
\eta_{11} & 0 & 0 \\
0 & \eta_{11} & 0 \\
0 & 0 & \eta_{33}
\end{array}\right), \\
& d=\left(\begin{array}{cccccc}
0 & 0 & 0 & 0 & d_{15} & -d_{22} \\
-d_{22} & d_{22} & 0 & d_{15} & 0 & 0 \\
d_{31} & d_{31} & d_{33} & 0 & 0 & 0
\end{array}\right)
\end{aligned}
$$

The coefficients shown inside the matrices of piezoelectric constants are termed as material constants [18]. 


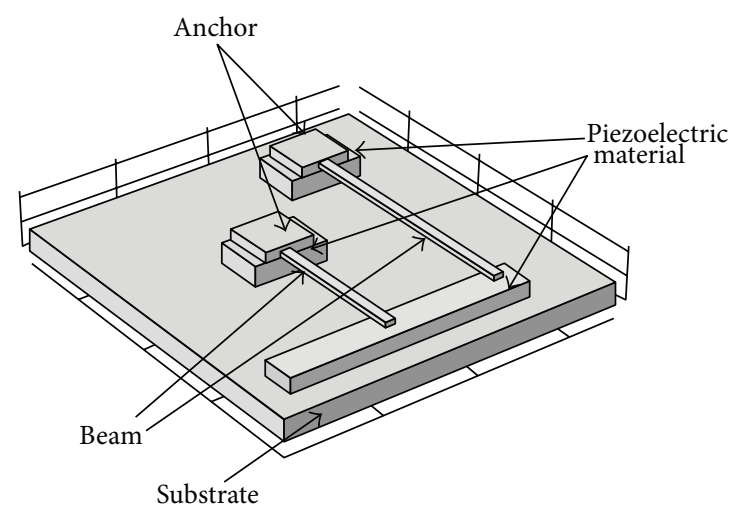

FIGURE 2: Layout of simulated cantilever design.

\section{Design Formation}

The model was designed using FEM supported COMSOL Multiphysics software in piezoelectric domain solver. Two microcantilevers with different dimensions are designed on polysilicon substrate. Figure 2 shows the geometry of our device. It consists of two microbeams connected to the same base. The vertical displacement of each cantilever can be independently controlled by applying a voltage on the beam. Though each cantilever is mechanically independent, however, the output depends on the vibrations of both cantilevers. The electrostatic force $F_{e}$ produced on the microbeam is proportional to the voltage applied on the microbeams.

The dimensions and the type of material used for designing the microcantilever are shown in Table 1, while the material properties selected are shown in Table 2 . Both cantilever beams are actuated by a piezoelectric base/anchor shaker. For low frequency generator design, the main emphasis is to produce the low resultant frequency at the output electrode. Besides the properties shown in Table 2, the piezoelectric material also depends on some other parameters that are equally responsible for the successful design of a microcantilever. Table 3 shows the values of different material constants used during device simulation.

The electrical boundary condition was applied to stimulate the piezoelectric material. Two different analytic signals were applied to the side boundaries of a piezoelectric material and the opposite boundary of the substrate is grounded. The input frequencies are selected randomly, but care has been taken that the selected applied frequencies should be high and must satisfy (5). The analytic signal applied to smaller cantilever is

$$
\text { an_signal }=5 \sin \left(2 \pi f_{1}\right) t \text {, }
$$

where $f_{1}$ is $10 \mathrm{MHz}$. The waveform is shown in Figure 3.

Similarly for longer cantilever, the applied signal is an_signal $=5 \cos \left(2 \pi f_{2}\right) t$.

The applied frequency $f_{2}$ is $11 \mathrm{MHz}$ and is shown in Figure 4 . From these equations, it is clear that the potential is set to +5 volts for both beams.

Theoretically, the solution to find the cantilever deflection $(\delta)$ is a function of the applied voltage; that is, with

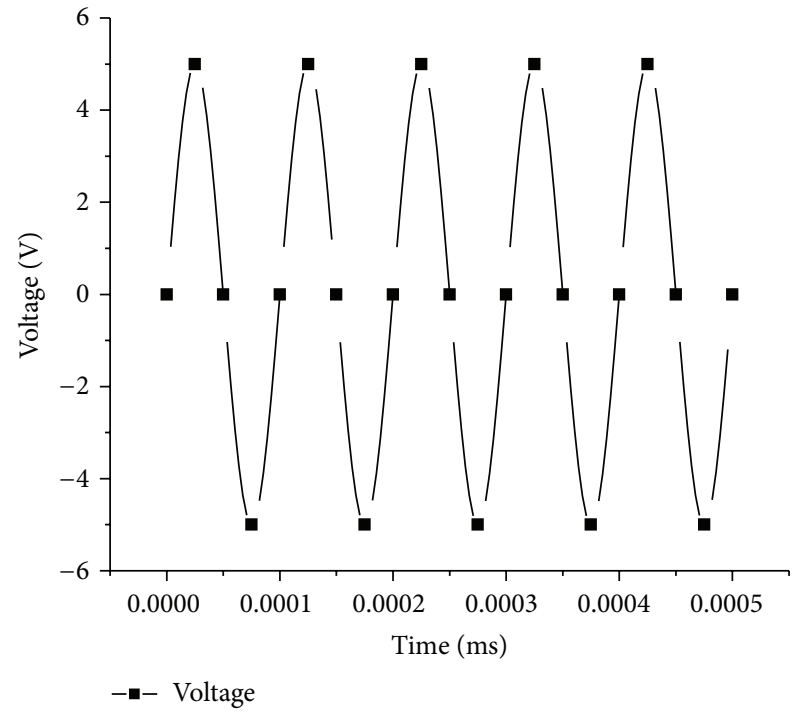

FIGURE 3: Input sinusoidal waveform applied to smaller cantilever beam.

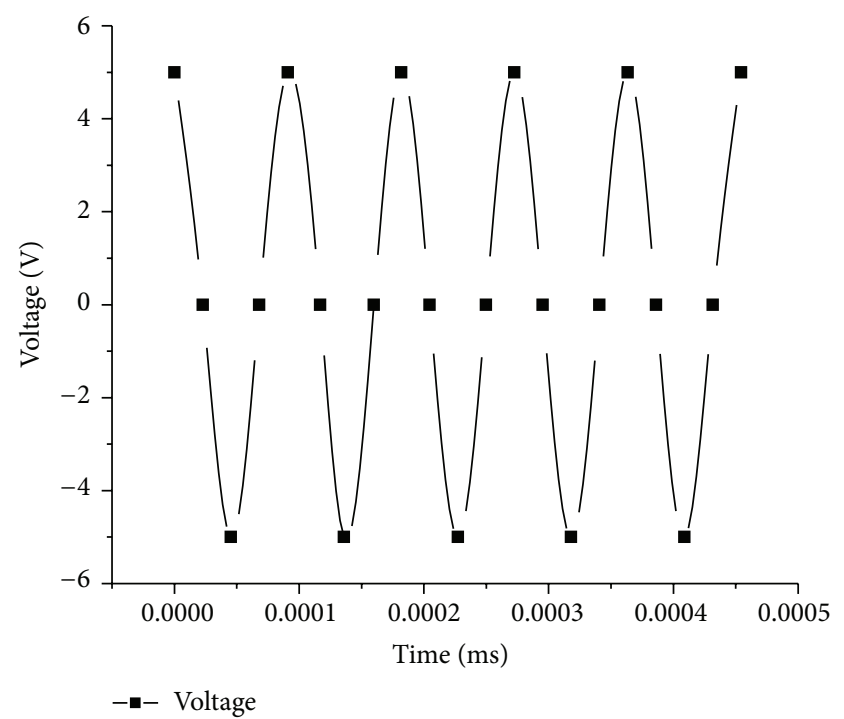

FIGURE 4: Input cosine waveform applied to longer cantilever beam.

the support of nonlinear differential equation, the deflection can be found out. The maximum voltage that can be applied to observe the deflection of the beam is the snap-in or collapse voltage. Above this voltage, structural defects or the cracks/fractures in the beam will take place and the cantilever may crumple, and this condition is irretrievable. The snap-in voltage can be calculated by finding the first derivative of the total potential energy.

\section{Results and Discussions}

Results are evaluated with $3 \mathrm{D}$ finite element simulation using COMSOL 4.3. Figure 5 shows the design of the structure after meshing. Extrafine meshing is applied on the entire 
TABLE 1: Materials and dimensions.

\begin{tabular}{lcccc}
\hline Parameters & Substrate & Piezoelectric material & Beam material 1 & Beam material 2 \\
\hline Material & Silicon & Lead zirconate titanate (PZT-5H) & Aluminium & 35 \\
Length & 75 & 10 & 2 & Aluminium \\
Width & 70 & 15 & 1 & 2 \\
Height & 5 & 4 & 1 & 1 \\
\hline
\end{tabular}

TABLE 2: Material properties.

\begin{tabular}{lccc}
\hline Coefficients & Silicon & Aluminium & PZT-5H \\
\hline Density & 2330 & 2700 & 7600 \\
Young's modulus $(\mathrm{Pa})$ & $170 \times 10^{9}$ & $70 \times 10^{9}$ & $65 \times 10^{9}$ \\
Poisson's ratio & 0.28 & 0.33 & 0.35 \\
\hline
\end{tabular}

TABLE 3: Material constants for the materials used in design.

\begin{tabular}{|c|c|c|c|c|c|c|c|c|c|c|c|c|}
\hline & \multicolumn{12}{|c|}{ Material constants } \\
\hline & $S_{11}$ & $S_{12}$ & $S_{13}$ & $S_{14}$ & $S_{33}$ & $S_{44}$ & $\eta_{11}$ & $\eta_{33}$ & $d_{15}$ & $d_{22}$ & $d_{31}$ & $d_{33}$ \\
\hline Units & $\mathrm{Nm}^{-2}$ & $\mathrm{Nm}^{-2}$ & $\mathrm{Nm}^{-2}$ & $\mathrm{Nm}^{-2}$ & $\mathrm{Nm}^{-2}$ & $\mathrm{Nm}^{-2}$ & $\mathrm{Fm}^{-1}$ & $\mathrm{Fm}^{-1}$ & $\mathrm{Cm}^{-2}$ & $\mathrm{Cm}^{-2}$ & $\mathrm{Cm}^{-2}$ & $\mathrm{Cm}^{-2}$ \\
\hline Silicon & $203 \times 10^{9}$ & $530 \times 10^{9}$ & $750 \times 10^{9}$ & $90 \times 10^{9}$ & $245 \times 10^{9}$ & $60 \times 10^{9}$ & 44 & 29 & 3.7 & 2.5 & 0.2 & 1.3 \\
\hline Aluminium & $106 \times 10^{9}$ & $60 \times 10^{9}$ & $106 \times 10^{9}$ & $59 \times 10^{9}$ & $25 \times 10^{9}$ & $25 \times 10^{9}$ & 2 & 2 & 0 & 0 & 0 & 0 \\
\hline PZT-5H & $126 \times 10^{9}$ & $800 \times 10^{9}$ & $840 \times 10^{9}$ & 0 & $120 \times 10^{9}$ & $23 \times 10^{9}$ & 1500 & 10 & 17 & 0 & 65 & 233 \\
\hline
\end{tabular}

model. The maximum element size selected is 8 . The design is simulated on the computational machine with $3.6 \mathrm{GHz}$ processor speed. The virtual memory used while simulation is $2.9 \mathrm{~GB}$. Extremely fine meshing is not selected to avoid the computational load. Time dependent settings is selected for simulating the model the study of the simulated model. For smooth outcomes of the results, the model is simulated for the time period 0 to $1 \mu \mathrm{s}$.

The schematic of a simulated device as low frequency generator is shown in Figure 6.

The output generated waveform due to input analytic signals is shown in Figure 7. This shows the deformation of the tip of the free end of the beam. Due to deformation, the tips of the beam touch the piezoelectric material which in turn generates the resultant frequency due to vibratory movements of both beams. The frequency versus voltage graph indicates that there is a change in displacement of the free end of the microcantilever beam, and hence the resultant plot shows the one complete cycle of the sinusoidal waveform in almost $117 \mathrm{KHz}$ that comes under the low frequency (LF) region of electromagnetic spectrum.

Figure 8 shows the plot for the total displacement with respect to the frequency. The graph clearly shows that the beam gets displaced from its mean position (static state) to the extreme position and slowly returns back to its mean position. However, number of vibrations depends on the time dependent settings of the model.

Figure 9 illustrates the graph for the mean stress taken at the microscale at a particular location in a model. This plot shows that the mean or average stress on the beam changes in accordance with the instantaneous position of the free end of the beams. Hence, this plot shows the mean stress levels at different time intervals. From the analysis, it is clear that

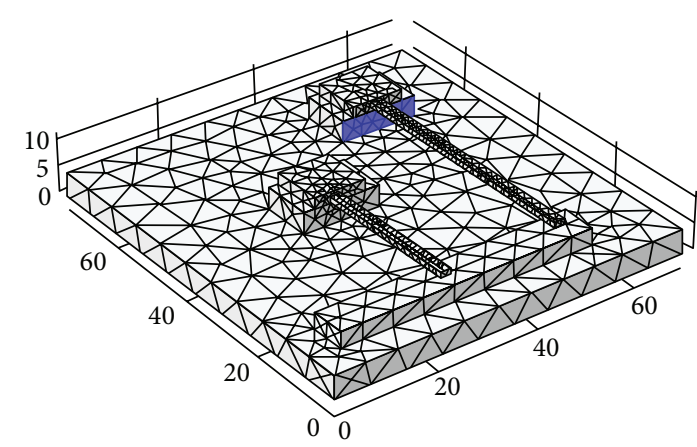

Figure 5: Schematic of the device after meshing.

higher values of mean stress at the microlevel are generated in different locations of the macrostructure.

\section{Conclusion}

In this work, the geometrical dimensions of piezoelectric microcantilever are analyzed using finite element analysis technique to obtain optimal performance as a low frequency generator. The aim of this work was to obtain the beat frequency (favourably a low frequency signal) using two different high frequency signals as inputs. The results obtained validate that the low frequency has been generated. However, the waveform is not an ideal sinusoidal signal but is little distorted. The distortion may be there due to some boundary and scattering losses. Our future work will be to reduce these losses and to generate optimum beat frequency. 


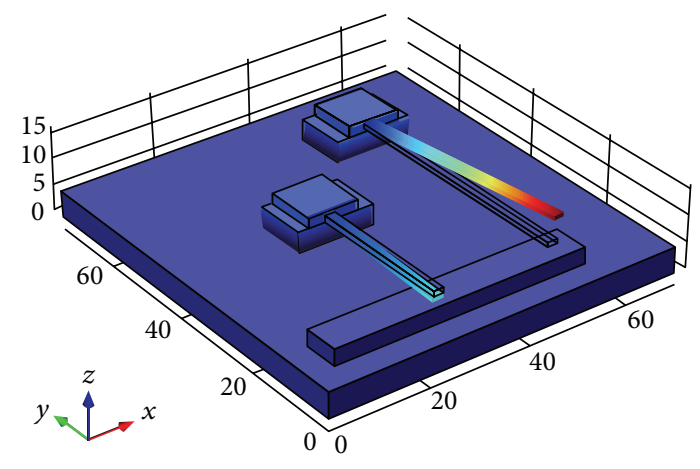

FIGURE 6: Schematic of a complete simulated device.

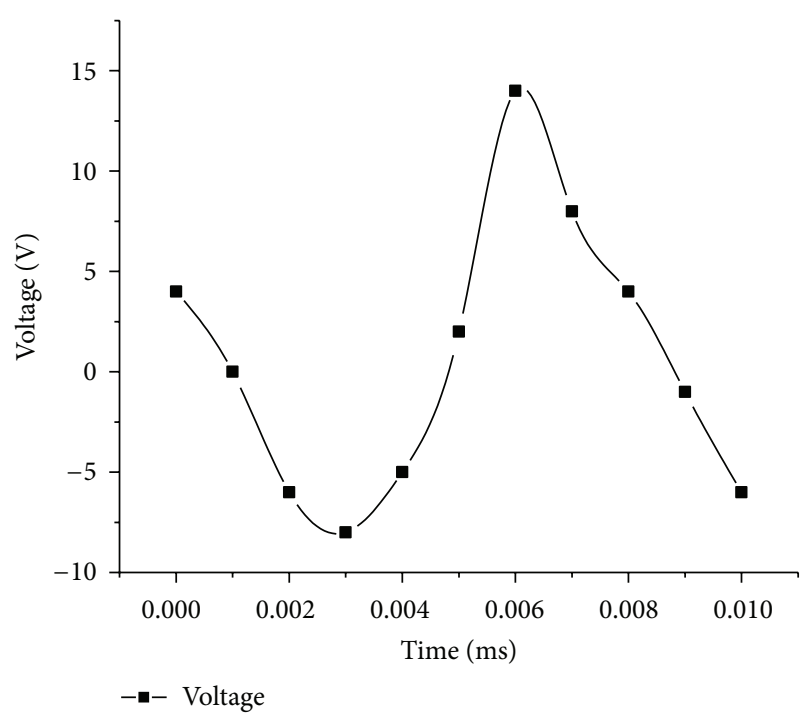

FIGURE 7: Resultant output waveform showing low frequency signal.

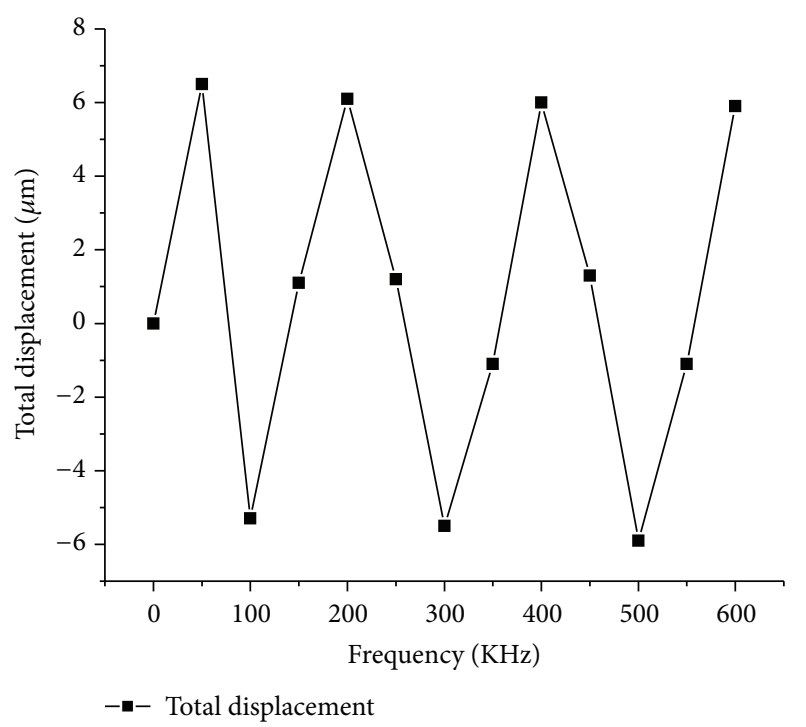

FIgURE 8: Plot of change in displacement with respect to frequency.

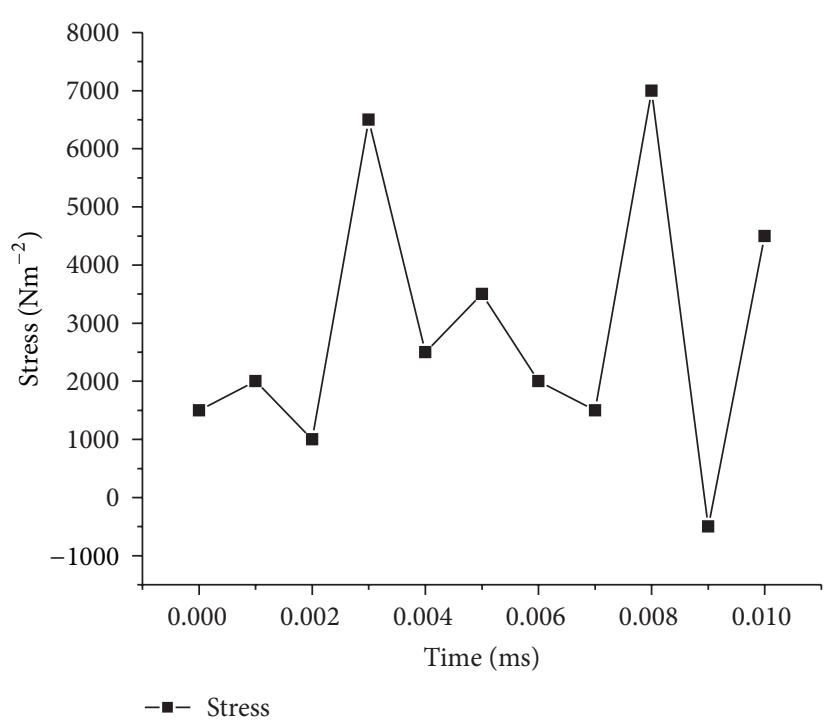

FIGURE 9: Surface stress on cantilever beams at different time intervals.

\section{Conflict of Interests}

This research work has been done using data gathered for carrying out the first author's Ph.D. related experiment and it is claimed that no funding or financial support from fellowship, scholarship, or any sponsors has been gained by the author. Therefore, the authors declare that there is no conflict of interests regarding the publication of this paper.

\section{References}

[1] H.-K. Liu, C. H. Pan, and P.-P. Liu, "Dimension effect on mechanical behavior of silicon micro-cantilever beams," Measurement, vol. 41, no. 8, pp. 885-895, 2008.

[2] A. Boisen, S. Dohn, S. S. Keller, S. Schmid, and M. Tenje, "Cantilever-like micromechanical sensors," Reports on Progress in Physics, vol. 74, no. 3, Article ID 036101, 2011.

[3] R. Raiteri, M. Grattarola, H.-J. Butt, and P. Skládal, "Micromechanical cantilever-based biosensors," Sensors and Actuators, B: Chemical, vol. 79, no. 2-3, pp. 115-126, 2001.

[4] R. Herdier, D. Jenkins, D. Remiens, M. Dupont, and D. Osmont, "A silicon cantilever beam structure for the evaluation of $\mathrm{d} 31$, d33 and e31 piezoelectric coefficients of PZT thin films," in Proceedings of the 16th IEEE International Symposium on the Applications of Ferroelectrics (ISAF '07), pp. 725-727, May 2007.

[5] X. Li and D.-W. Lee, "Integrated microcantilevers for highresolution sensing and probing," Measurement Science and Technology, vol. 23, no. 2, Article ID 022001, 2012.

[6] H. H. Yoo and S. H. Shin, "Vibration analysis of rotating cantilever beams," Journal of Sound and Vibration, vol. 212, no. 5, pp. 807-808, 1998.

[7] J. Thaysen, A. Boisen, O. Hansen, and S. Bouwstra, "Atomic force microscopy probe with piezoresistive read-out and a highly symmetrical Wheatstone bridge arrangement," Sensors and Actuators, A: Physical, vol. 83, no. 1, pp. 47-53, 2000.

[8] L. A. Pinnaduwage, A. Gehl, D. L. Hedden et al., "A microsensor for trinitrotoluene vapour," Nature, vol. 425, no. 6957, p. 474, 2003. 
[9] S. Arya, S. Khan, and P. Lehana, "Finite element modelling of piezoelectric micro-cantilever as gas sensor," in Proceedings of the 1st International Conference on Emerging Trends and Applications in Computer Science (ICETACS '13), 2013.

[10] B. Rogers, L. Manning, M. Jones et al., "Mercury vapor detection with a self-sensing, resonating piezoelectric cantilever," Review of Scientific Instruments, vol. 74, no. 11, pp. 4899-4901, 2003.

[11] S. Dohn, R. Sandberg, W. Svendsen, and A. Boisen, "Enhanced functionality of cantilever based mass sensors using higher modes," Applied Physics Letters, vol. 86, no. 23, Article ID 233501, pp. 1-3, 2005.

[12] H. Irschik, "A review on static and dynamic shape control of structures by piezoelectric actuation," Engineering Structures, vol. 24, no. 1, pp. 5-11, 2002.

[13] A. T. Tran, G. Pandrauda, H. Schellevisa et al., "Fabrication of AlN slender piezoelectric cantilevers for high speed MEMS actuations," Procedia Engineering, vol. 25, pp. 673-676, 2011.

[14] T. W. Kenny, S. B. Waltman, J. K. Reynolds, and W. J. Kaiser, "Micromachined silicon tunnel sensor for motion detection," Applied Physics Letters, vol. 58, no. 1, pp. 100-102, 1991.

[15] S.-H. S. Lim, D. Raorane, S. Satyanarayana, and A. Majumdar, "Nano-chemo-mechanical sensor array platform for highthroughput chemical analysis," Sensors and Actuators, B: Chemical, vol. 119, no. 2, pp. 466-474, 2006.

[16] A. J. Qavi, A. L. Washburn, J.-Y. Byeon, and R. C. Bailey, "Labelfree technologies for quantitative multiparameter biological analysis," Analytical and Bioanalytical Chemistry, vol. 394, no. 1, pp. 121-135, 2009.

[17] J. Fritz, “Cantilever biosensors," Analyst, vol. 133, no. 7, pp. 855863, 2008.

[18] Y. Rao and G. Zhang, "3D modeling of a surface-acousticwave based sensor," in Proceedings of the COMSOL Conference, Boston, Mass, USA, 2007.

[19] J. Wang, J.-K. Chen, and S. Liao, "An explicit solution of the large deformation of a cantilever beam under point load at the free tip," Journal of Computational and Applied Mathematics, vol. 212, no. 2, pp. 320-330, 2008.

[20] I. Kim, H. Joo, S. Jeong, M. Kim, and J. Song, "Micro power generation of a PMN-PZT triple-morph cantilever for electric harvesting devices," Journal of the Korean Physical Society, vol. 56, no. 12, pp. 370-373, 2010. 

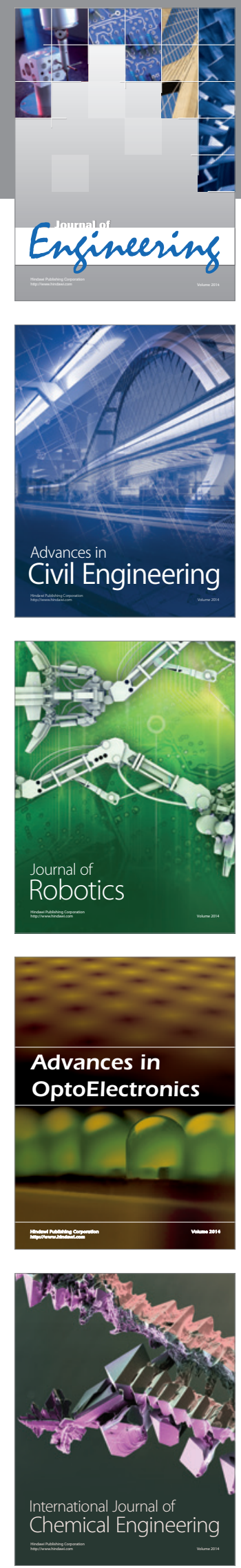

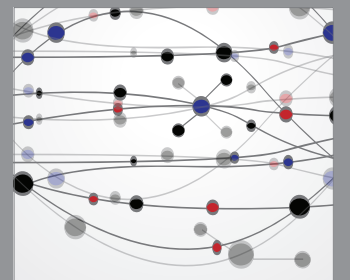

The Scientific World Journal
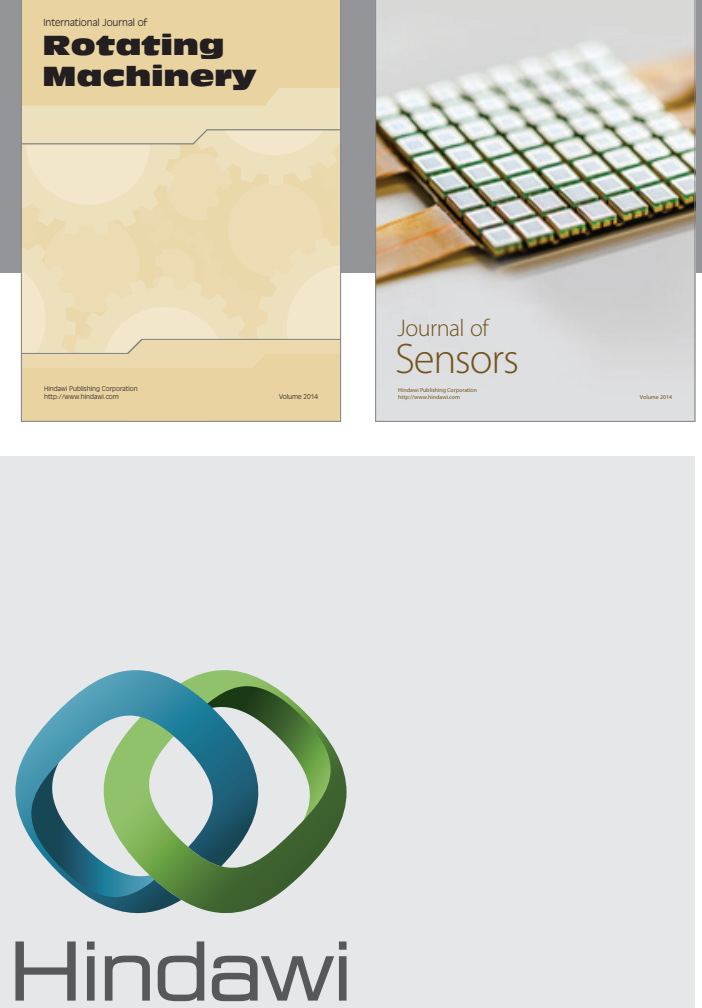

Submit your manuscripts at http://www.hindawi.com
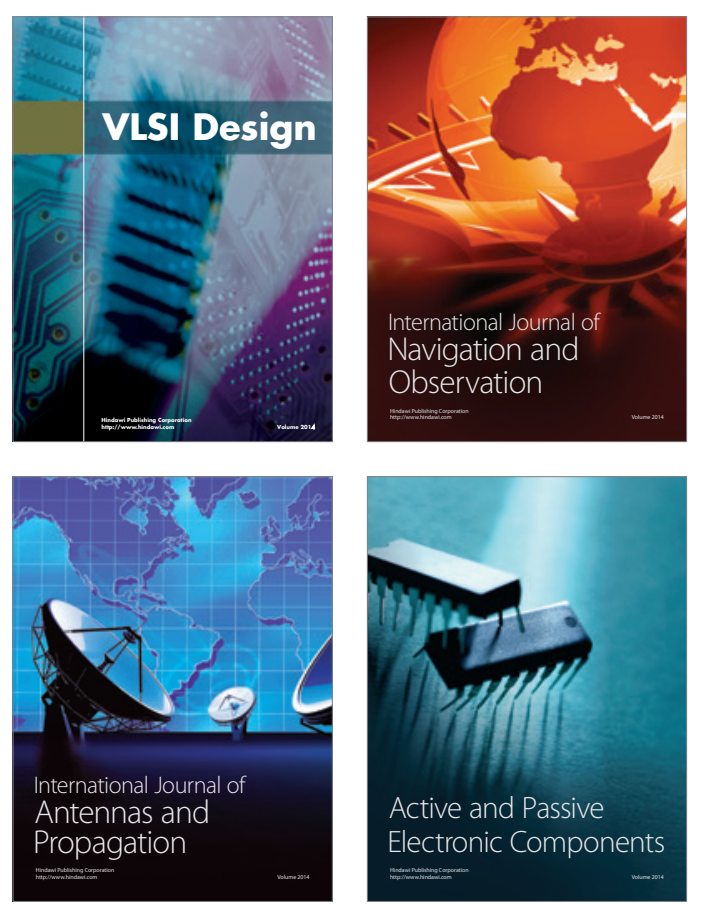
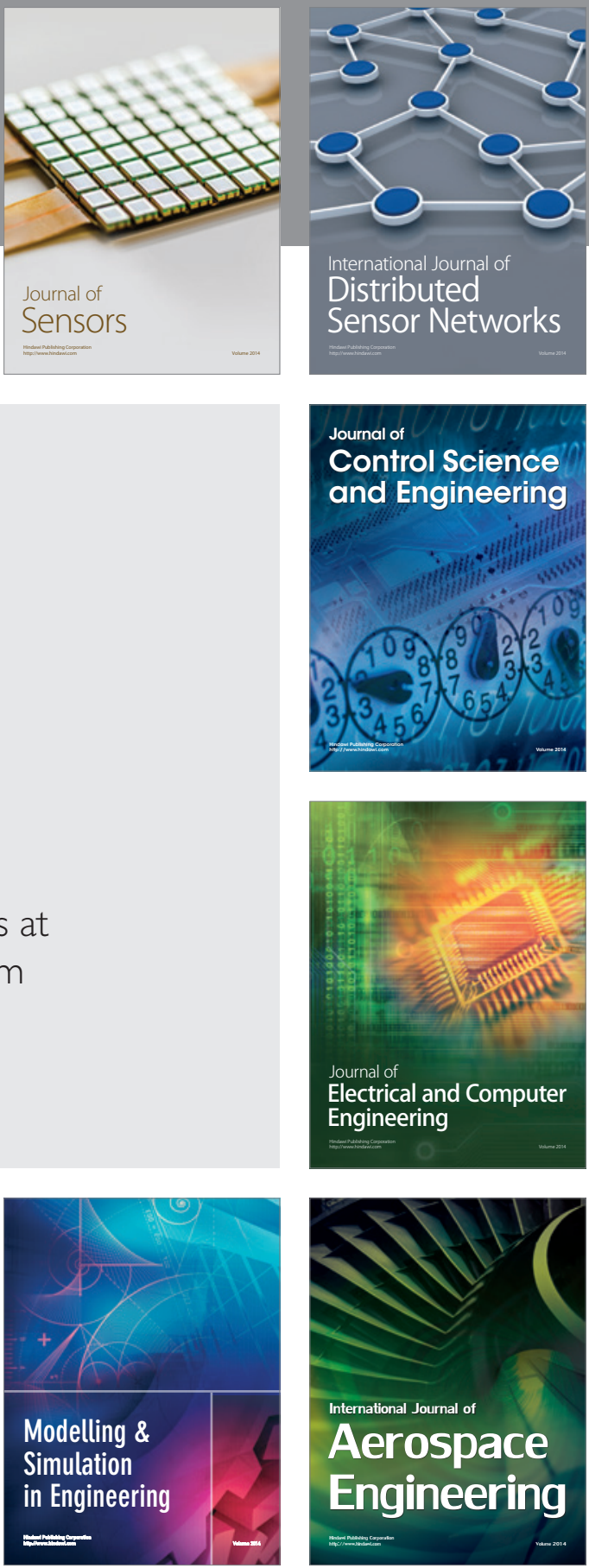

Journal of

Control Science

and Engineering
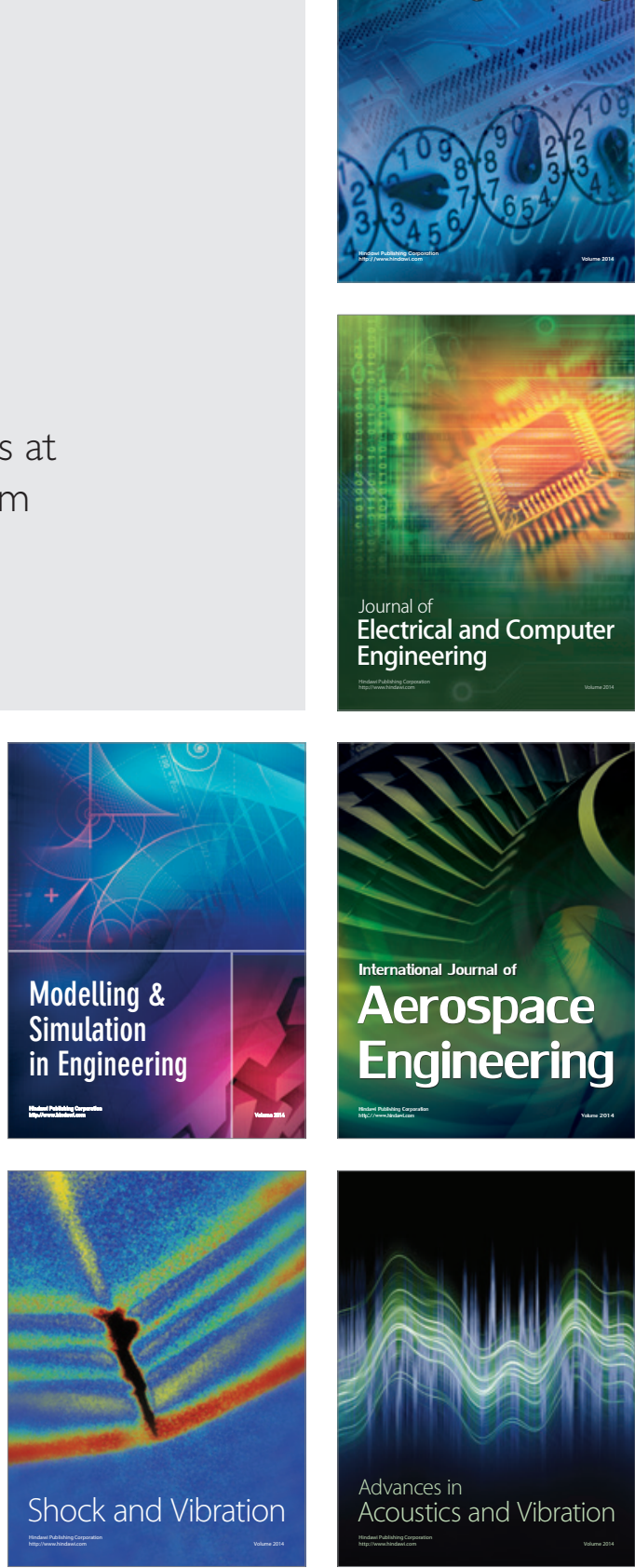